\title{
The rheological properties of blood and the risk of cardiovascular disease in patients with obstructive sleep apnea syndrome (OSAS)
}

\author{
Maciej Tażbirek ${ }^{1}$, Ludmiła Słowińska ${ }^{2}$, Marcin Kawalski ${ }^{3}$, Władysław Pierzchała ${ }^{1}$ \\ ${ }^{1}$ Department of Pulmonology, Medical University of Silesia in Katowice, Poland \\ ${ }^{2}$ Department of Biophysics, Medical University of Silesia in Zabrze, Poland \\ ${ }^{3}$ I. Moscicki Hospital in Chorzow, Poland
}

\begin{abstract}
Obstructive sleep apnea (OSA) is an important public health concern, which affects around 2-4\% of the population. Left untreated, it causes a decrease not only in quality of life, but also of life expectancy. Despite the fact that knowledge about the mechanisms of development of cardiovascular disease in patients with OSA is still incomplete, observations confirm a relationship between sleep disordered breathing and the rheological properties of blood. One possible consequence of an increased incidence of cardiovascular disease may be a rise in mortality in OSA patients. Continuously improved research methods are allowing for an increasingly more accurate understanding of the significance of observed changes. (Folia Histochemica et Cytobiologica 2011, Vol. 49, No. 2, 206-210)
\end{abstract}

Key words: obstructive sleep apnea, hemorheology, blood viscosity, erythrocyte deformability, cardiovascular diseases

Obstructive sleep apnea (OSA) is an important public health concern. The estimated prevalence is $2 \%$ in women and $4 \%$ in men [1]. It is characterized by recurrent episodes of upper airway restriction during sleep associated with an increased respiratory effort, periodic arterial oxygen desaturations and sleep structure disorders. Daytime symptoms include an insatiable sleepiness even after prolonged sleep, which usually is not restorative.

According to the American Academy of Sleep Medicine guidelines, obstructive sleep apnea can be diagnosed when respiratory disturbance index (RDI) is equal to or greater than 15 , or when it is greater than 5 and accompanied by one of the typical symptoms (pathological daytime sleepiness, restless sleep, fatigue or insomnia, awakenings with a feeling of choking, irregular snoring and/or pauses in breathing during sleep

Correspondence address: M. Tażbirek,

Department of Pulmonology,

Medical University of Silesia in Katowice,

Medyków Str. 14, 40-752 Katowice, Poland;

e-mail: tazbirek@gmail.com observed by bed partners) [2]. Full overnight polysomnography remains the gold standard of OSA diagnosis. The first line of treatment in OSA patients without significant comorbidities in the anatomy of their upper respiratory tract is the use of positive airway pressure (CPAP, continuous positive air pressure).

Current knowledge regarding the natural history of OSA is incomplete, but both the immediate and the long-term consequences of untreated OSA are undeniable [1, 3-6]. Circadian distribution of cardiovascular events including heart attacks and strokes suggests an interaction between awakenings and acute thrombosis.

It is believed that the increased incidence of vascular events during the morning hours is linked in some cases with coexisting OSA [5]. Such observations suggest the need for further research into the role of sleep and associated disorders in the pathogenesis of cardiovascular system diseases. Observations confirm a relationship between cardiovascular incidents and sleep breathing disorders, both acute (e.g. stroke, myocardial infarction, sudden death during sleep) and chronic (e.g. hypertension, ischemic 
heart disease, heart failure) [1]. Due to the number of common risk factors, this relationship cannot be viewed as a simple cause and effect relationship.

The pathogenesis of cardiovascular disease in patients with sleep apnea syndrome undoubtedly depends on several factors. One important association between OSA and cardiovascular disease is excessive sympathetic nervous system activity. Platelet aggregation increase in patients with OSA may be secondary to the increased nocturnal levels of catecholamines. The elimination of apnea incidents via CPAP therapy reduces platelet aggregation in conjunction with nocturnal levels of catecholamines. Along with the prevailing sympathetic activation, endothelial dysfunction has been reported as one cause of cardiovascular disease. Hypoxia, hypercapnia, and blood pressure increases associated with OSA are elements of endothelial function impairment. Increased levels of endothelines may contribute to vasoconstriction and other changes in the cardiovascular system.

Episodes of hypoxia followed by re-oxygenation occur consecutively many times during the night over many years in patients with untreated severe OSA, and this leads to oxidative stress. The combination of hypoxemia and sleep deprivation found in patients with OSA also increases levels of inflammatory markers $[1,7]$. Increased blood clotting, caused by changes in the rheological properties of blood and plasma $[1,3]$, seems to be an important factor linking OSA and cardiovascular complications.

Understanding the physical phenomena associated with blood flow has been the subject of many recent studies [5, 8-12]. The field of science devoted to the analysis of these phenomena is called hemorheology. Evaluation of the rheological properties of blood is based on measuring the viscosity of whole blood and plasma and determining erythrocytes aggregation and deformation abilities. Viscosity (viscosity coefficient) is a measure of the resistance of the liquids with mutual displacement of the fluid layers. It is determined by the ratio of shear stress to shear rate. Tangential stress $\gamma$ is defined as the ratio of tangential force to the surface of the layers of flowing liquid. Shear rate $\mathrm{g}$ is the velocity gradient between the layers of the liquid [8]. At constant temperature and pressure, the viscosity of Newtonian fluids also remains constant, whereas in the case of non-Newtonian fluids it is a function of shear rate. Whole blood viscosity measurements are carried out using capillary rheometers or plasma-rotational rheometers, which also allow the measurement of plasma viscosity. The ability of erythrocytes to aggregate is determined in aggregometers by measuring the scattered light. Erythrocyte deformability is assessed on the

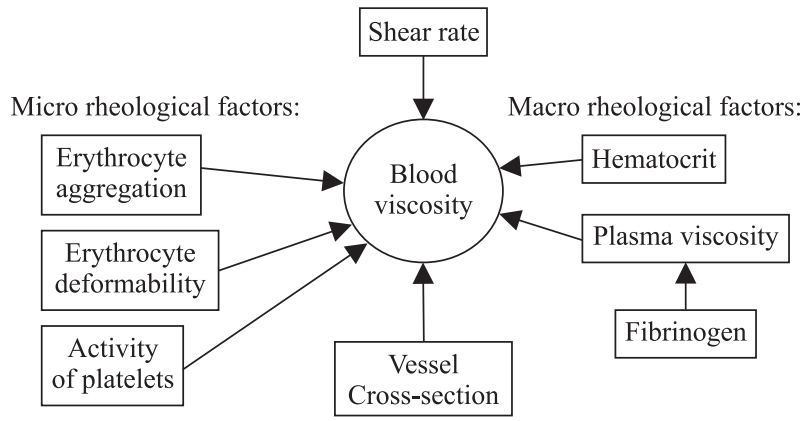

Figure 1. Determinants of blood viscosity at stable temperature

basis of flow time, either through the capillary or filtration methods.

Blood is a liquid constituting a heterogeneous cell suspension in the plasma. It is a typical non-Newtonian fluid of pseudo-plastic and thixotropic characteristics with complex rheological properties. Liquids in which viscosity decreases monotonically with the increase of shear rate, are called 'pseudo-plastic liquids'. Non-Newtonian liquids, where viscosity depends not only on shear rate, but also on the time and manner of shearing, are called 'thixotropic liquids' [9]. Thixotropic properties are associated with the formation of structures in the absence of shear, and breakdowns in the presence of shear stress [9]. The main factors determining the viscosity of blood are temperature, vessel cross-section and shear rate factors such as hematocrit, plasma viscosity, erythrocyte aggregation and deformability [5], and the activity of platelets. Hematocrit and plasma viscosity are socalled 'macro rheological' factors, whereas deformability and aggregation are called 'micro rheological' factors (Figure 1). The pathogenetic role of these factors varies. The relationship between hematocrit and viscosity is non-linear. The effect of the plasma on the viscosity of the blood is complex, and is both direct and indirect [13]. The indirect effect is associated with the presence of fibrinogen and a2-globulin. The direct effect is related to the flow of the erythrocytes suspended in plasma adjacent to the vascular endothelium in narrow capillaries. The increase in plasma viscosity impedes the flow, and thus deteriorates tissue perfusion [10]. Increased plasma viscosity also plays an important role in platelet adhesion to the vascular endothelium. At constant hematocrit and low shear rates, increased erythrocyte aggregation is a major factor affecting blood viscosity, whereas at high shear rates the major factor is a reduced deformability [9]. Erythrocyte aggregation plays a significant role in parts of the circulatory system, where blood flow velocity gradients are reduced. 
Blood viscosity is also determined by the mechanical properties of red blood cells, mainly their deformability. It involves a special ability of shape shifting of the red blood cells while maintaining an unchanged volume. Such properties are of particular importance during flow of the erythrocytes in the capillaries, the diameter of which is smaller than that of the red blood cells.

Although many papers on OSA and its traditional cardiovascular risk factors have been published, few publications have taken into account the role of blood coagulation [2] and its influence on the rheological properties of blood [14-19]. The limited number of publications and methodological differences among studies that describe the rheological properties of blood hinder their comparative analysis. Lack of consistent definitions for the diagnosis of sleep apnea is also a limiting factor. The pioneering work of Chin et al. [14] confirmed in 11 patients increased levels of fibrinogen, hematocrit and indirectly blood viscosity in the morning. An AHI of 20 (20 apnea/hypopnea episodes per hour) and the presence of clinical symptoms were used as study inclusion criteria.

Nobili et al. [15] showed that blood viscosity increased in the mornings in a group of 12 patients with OSAS, but not in a group of eight healthy controls. The criterion for diagnosis was respiratory distress index $>5 /$ h confirmed by a polysomnography study and the presence of clinical symptoms.

Reinhard et al. [16] described the impact of six-month CPAP therapy on a group of 13 patients with OSA compared to eight healthy controls. They found that morning plasma viscosity was higher in patients with OSA. Fibrinogen levels showed a correlation with plasma viscosity. They also confirmed that blood viscosity and platelet activity in patients treated with CPAP was comparable to healthy controls, and that a single night without using a CPAP device had no significant effect on examined blood parameters.

Zhang et al. [17] evaluated the biochemical characteristics of a prothrombotic state in a group of 41 elderly patients (mean age $63.4 \pm 4$ years) with polysomnographically diagnosed sleep apnea syndrome on the basis of AHI $\geq 5 / \mathrm{h}$ before and after 30-day CPAP therapy, and compared the results to those of healthy controls matched for age. They confirmed a statistically significant increase, in the group with OSA, of hematocrit, blood viscosity and platelet aggregation. The 30-day CPAP therapy resulted in morning reduction of hematocrit, total blood viscosity, platelet aggregation and prolongation of the prothrombin time and activated partial thromboplastin time.

Tażbirek et al. [18] found an increase in blood viscosity, corrected blood viscosity, plasma viscosity and erythrocyte aggregation in obese men with polysomnographically confirmed OSA (AHI 45 with an average rate of 16.2 episodes per hour) as compared to patients without OSA, matched in terms of age, BMI and the incidence of coexisting diseases. They showed that there was a correlation between the values of measured parameters before the start of CPAP therapy (blood viscosity, corrected blood viscosity, plasma viscosity, aggregation index) in patients with OSA and the polysomnographic severity indicators of OSA (apnea-hypopnea index, $\mathrm{SaO}_{2}$ min, $\mathrm{SaO}_{2}<90 \%$ ).

The primary determinant of blood viscosity is the viscosity of plasma. Increased viscosity affects the frictional forces that inhibit the movement of erythrocytes; it positively correlates with total cholesterol [21], low-density lipoproteins (LDL) and triglycerides $[19,22]$. Also, correlations between increased levels of fibrinogen and plasma viscosity in obese patients [23], as well as between fibrinogen and ischemic heart disease, have been confirmed [24].

Wessendorf et al. [20] showed that elevated plasma fibrinogen levels in stroke patients are accompanied by a higher incidence of OSA. Their data confirmed a positive correlation between the plasma fibrinogen concentration and the number and duration of recorded breathing disorders during sleep, and a negative correlation with minimum and average blood saturation. The authors suggest that high concentrations of fibrinogen may lead to an increased incidence of vascular disease in OSA.

Dikmenoglu et al. [5] compared the rheological properties of blood in 11 patients with severe sleep apnea syndrome to a control group matched for age and gender. They found that plasma viscosity in patients with severe OSA was significantly higher. Morning blood viscosity showed a negative correlation with the average and minimum saturation levels, and a positive correlation with time spent in hypoxia $\left(\mathrm{SaO}_{2}<\right.$ $<90 \%$ ) [5]. Elevated levels of proteins, including biochemical markers of inflammation, are indicated as a possible mechanism of increased plasma viscosity in patients with OSA. A relationship between sleep disordered breathing and increased levels of CRP and IL6 has been confirmed, as well as a correlation with the severity of OSA [25-27]. Treatment with CPAP, according to some authors, influences the decrease of these markers [25] although other researchers have cast doubt on that dependency [28, 29]. It seems that an important element linking inflammation with vascular damage and sleep disordered breathing is the phenomenon of periodic hypoxia followed by re-oxygenation. Dikmenoglu et al. [5] described increased morning levels of plasma malondialdehyde (malondialdehyde-MDA) in a group of patients with severe 
OSA. Malondialdehyde is a product of per-oxidation of polyunsaturated fatty acids and is one of the indicators of oxidative stress, which affects the properties of plasma proteins. Also, in an earlier study by Polish researchers [18], the increase in plasma viscosity in the group with OSA compared to controls was confirmed in the absence of statistically significant differences in the total level of proteins, CRP and fibrinogen. An increase of the aggregation index in the group with OSA [18] suggests that another possible explanation of the cause of increased plasma viscosity, which affects the ability of the erythrocytes to aggregate, may be the change of the connections among the proteins in plasma.

Aggregation is a complex phenomenon, which arises from the combined effects of both the cell membrane of erythrocytes (the electric charge, the viscoelastic properties of the cell membrane) and plasma factors. Plasma factors which specifically increase erythrocyte aggregation are known as 'bridging proteins'. Examples are fibrinogen, and 2-globulin and immunoglobulins.

Increased erythrocyte aggregation ability within a short period, and the statistically significant effects of just five days of CPAP therapy on the parameters of erythrocyte aggregation, point to the important contribution of erythrocyte aggregation on the rheological properties of blood in patients with OSA [18]. Significant correlations between aggregation parameters (aggregation index, time T 1/2) and the parameters describing polysomnographic severity (time spent in hypoxia- $\mathrm{SaO}_{2}<90 \%$, index of apnea and hypopnea counts breathing-AHI and the apnea length) confirm this relationship.

Deformability of erythrocytes is yet another important factor in blood viscosity: a reduction in deformability increases blood viscosity $[11,30]$. In the phenomenon of deformation, it is primarily the cell membrane and cytoskeleton proteins that are involved.

Increased activity of antioxidant enzymes, which is observed in patients with OSA, supports the intensification of per-oxidation. Malondialdehyde (MDA), which is the product of per-oxidation, modifies the physical properties of cell membranes, causing disruptions in the hydrophobicity of the lipid interior and the violation of the bilayer membrane structure [31]. Lipid per-oxidation and its by-products cause polymerization of membrane components and lead to an increase in the stiffness of the phospholipid bilayer [32]. Yet there has been no unequivocal data on impaired erythrocyte deformability as the main mechanism of increased blood viscosity. Previous studies $[5,18]$ show only a statistically insignificant difference between patients with OSA and controls. Failure to find the impact of a five-day CPAP therapy [18] on the test indicators of deformability justifies the need for future assessment of the impact of long-term therapy.

One key rheological property of blood is the platelet function. Published studies have shown that platelet aggregation is increased in patients with OSA and may be secondary to the increased levels of catecholamines during the night [1]. The release of catecholamines which accompanies awakenings [32] may contribute to the change of rheological properties. Decrease of the number of apnea and hypopnea episodes to the level of physiological breathing with the aid of CPAP reduces the ability of platelet aggregation in conjunction with the change in levels of catecholamines [33, 34].

Hui et al. [35] confirmed increased platelet activation in a study of 42 patients with OSA (AHI $>10$ / /hr.) compared to a control group of healthy subjects matched for body weight. Platelet activation in that study correlated with the rate of awakenings. CPAP treatment reduced the activity of platelets, suggesting that it may have a cardioprotective effect.

Although knowledge about the mechanisms of development of cardiovascular disease in patients with OSAS is still incomplete, current observations confirm a relationship between sleep disordered breathing and changes in the rheological properties of blood. The concomitant increase in the frequency of cardiovascular disease observed in this group may be a consequence of such changes. Continuously improved research methods are allowing for an increasingly more accurate understanding of the significance of observed changes.

\section{References}

1. McNicholas WT, Bonsignore MR; the Management Committee of EU COST ACTION B26. Sleep apnoea as an independent risk factor for cardiovascular disease: current evidence, basic mechanism and research priorities. Eur Respi J. 2007;29:156-178.

2. Jax S, Hennersdorf S, Schwalen A, Strauer B. Altered blood rheology in obstructive sleep apnea as a mediator of cardiovascular risk. Cardiology. 2005;104:92-96.

3. Shamsuzzaman AS, Gersh BJ, Somers VK. Obstructive sleep apnea: implications for cardiac and vascular disease. JAMA. 2003;290:1906-1914.

4. Tażbirek M, Pierzchała W. Consequences of untreated obstructive sleep apnoea syndrome. Fam Med Prim Care Rev. 2008;10:1106-1111.

5. Dikmenoglu N, Ciftci B, Ileri E et al. Erythrocyte deformability, plasma viscosity and oxidative status in patients with severe obstructive sleep apnea syndrome. Sleep Med. 2006;7: 255-261.

6. Labuz-Roszak B, Tażbirek M, Pierzchala K, Pierzchala W. Ocena częstości występowania zespołu bezdechu we śnie u chorych we wczesnej fazie udaru mózgu. Pol Merkur Lek. 2004:16:536-538. 
7. Garvey JF, Taylor CT, McNicholas WT. Cardiovascular disease in obstructive sleep apnoea syndrome: the role of intermittent hypoxia and infamation. Eur Respir J. 2009;33:1195-1205 .

8. Wawrzyniak E. Lepkość krwi - podstawowe pojęcia i zależności. Diagn Lab. 1996;32:495-502.

9. Turczynski B. Właściwości reologiczne krwi i ich rola. W: Jaroszyk F. (red.). Biofizyka. Warszawa: PZWL, 2001; 598-604.

10. Landgraf $\mathrm{H}$. Correlation between plasma viscosity and tissue oxygen tension. Clin Hemorheol Microcirc. 1999;20:37-40.

11. Chien S. Role of blood cells in microcirculatory regulation. Microvasc Res. 1985;29:129-151.

12. Clifford PS. Local control of blood flow. Adv Fizjolc Educ. 2011;35:5-15.

13. Turczyński B, Pierzchała K, Słowińska L, Becelewski J. Dynamika zmian właściwości reologicznych krwi i osocza w niedokrwiennym udarze mózgu. Wiad Lek. 2002;55:189-196.

14. Chin K, Ohi M, Kita $\mathrm{H}$ et al. Effect of NCPAP therapy on fibrinogen levels in obstructive sleep apnea syndrome. Am J Respir Crit Care Med. 1996;153:1972-1976.

15. Nobili L, Schiavi G, Bozano E, De Carli F, Ferrillo F, Nobili F. Morning increase of whole blood viscosity in obstructive sleep apnea syndrome. Clin Hemorheol Microcirc. 2000;22: 21-27.

16. Reinhart WH, Oswald J, Walter R, Kuhn M. Blood viscosity and platelet function in patients with obstructive sleep apnea syndrome treated with nasal continuous positive airway pressure. Clin Hemorheol Microcirc. 2002;27:201-207.

17. Zhang X, Yin K, Wang H, Su M, Yang Y. Effect of continuous positive airway pressure treatment on elderly Chinese patients with obstructive sleep apnea in the prothrombotic state Chin Med J (Eng). 2003;116:1426-1428.

18. Tazbirek M, Slowińska L, Skoczyński S, Pierzchała W. Short-term continuous positive airway pressure therapy reverses the pathological influence of obstructive sleep apnea on blood rheology parameters. Clin Hemorheol Microcirc. 2009; 41:241-249.

19. Lowe GD. Blood viscosity, lipoproteins and cardiovascular risk. Circulation. 1992;85:2329-2331.

20. Wessendorf TE, Thilmann AF, Wang YM. Fibrinogen levels and obstructive sleep apnea in ischemic stroke. Am J Respir Crit Care Med. 2000;162:2039-2042.

21. Lowe GD. Blood viscosity, lipoproteins and cardiovascular risk. Circulation. 1992;85:2325-2331.
22. De Simone G, Devereux RB, Chien S, Alderman MH, Atlas SA, Larach JH. Relation of blood viscosity to demographic and physiologic variables and to cardiovascular risk factors in apparently normal adults. Circulation. 1990;81:107-117.

23. Fanari P, Somazzi R, Nasrawi F et al. Haemorheological changes in obese adolescents after short-term diet. Int J Obes Relat Metab Disord. 1993;17:487-494.

24. Heinrich J, Balleisen L, Schulte H, Assmann G, Van deLoo $\mathrm{J}$. Fibrinogen and factor VII in the prediction of coronary risk. Results from the PROCAM study in healthy men. Arterioscler-Thromb. 1994;14:54-59.

25. Yokoc T, Minoguchi K, Matsuo $\mathrm{H}$ et al. Elevated levels of C-reactive protein and interleukin-6 in patients with obstructive sleep apnea syndrome and decreased by nasal continuous positive airway pressure. Circulation. 2003;107:1129-1134.

26. Tauman R, Ivanenko A, O'Brien LM, Gozal D. Plasma C-reactive protein levels among children with sleep disordered breathing. Pediatrics. 2004;113:e564-569.

27. Carpagnano GE, Kharitonov SA, Resta O, Foschino-Barbaro MP, Gramiccioni E, Barnes PJ. Increased 8-isoprostane and interleukin- 6 breath condensate of obstructive sleep apnea patients. Chest. 2002;122:1162-1167.

28. Guilleminault C, Kirisoglu C, Ohayon MM. C-reactive protein and sleep disordered breathing. Sleep. 2004;27:1507-1511.

29. Athanasios G, Kaditis AG, Alexopoulos EI et al. Morning levels of C-reactive protein in children with obstructive sleep-disordered breathing. Am J Respir Crit Care Med. 2005; 171:282-286.

30. Stuart J, Nash GB. Red cell deformability and hematological disorders. Blood Rev. 1990;4:141-147.

31. Gaweł S, Warda M, Niedworok E, Warda P. Dialdehyd malonowy (MDA) jako wskaźnik procesów peroskydacji lipidów w organizmie. Wiad Lek. 2004;LVII;453-455.

32. Coy TV, Dismsdale JE, Ancoli-Israel S, Clausen J. Sleep apnoea and sympathetic nervous system activity: a review. J Sleep Res. 1996;5:42-50.

33. Eisensehr I, Ehrenberg BL, Noachtar S et al. Platelet activation, epinephrine, and blood pressure in obstructive sleep apnea syndrome. Neurology. 1998;51:188-195.

34. Sanner BM, Konermann M, Tepel M, Groetz J, Mummenhoff C, Zidek W. Platelet functions in patients with obstructive sleep apnoea syndrome. Eur Respir J. 2000;109:562-567.

35. Hui DS, Ko FW, Fok JP et al. The effects of nasal CPAP on platelet activation in obstructive sleep apnea syndrome. Chest. 2004;125;1768-1775

Submitted: 15 February, 2011

Accepted after reviews: 1 May, 2011 